\title{
Hierarchical Distribution Matching for Probabilistically Shaped Coded Modulation
}

\author{
Tsuyoshi Yoshida, Member, IEEE, Magnus Karlsson, Fellow, OSA; Senior Member, IEEE, \\ and Erik Agrell, Fellow, IEEE
}

(Invited Paper)

\begin{abstract}
The implementation difficulties of combining distribution matching (DM) and dematching (invDM) for probabilistic shaping (PS) with soft-decision forward error correction (FEC) coding can be relaxed by reverse concatenation, for which the FEC coding and decoding lies inside the shaping algorithms. PS can seemingly achieve performance close to the Shannon limit, although there are practical implementation challenges that need to be carefully addressed. We propose a hierarchical DM (HiDM) scheme, having fully parallelized input/output interfaces and a pipelined architecture that can efficiently perform the DM/invDM without the complex operations of previously proposed methods such as constant composition DM (CCDM). Furthermore, HiDM can operate at a significantly larger post-FEC bit error rate (BER) for the same post-invDM BER performance, which facilitates simulations. These benefits come at the cost of a slightly larger rate loss and required signal-to-noise ratio at a given postFEC BER.
\end{abstract}

Index Terms-Bit error rate, block error rate, distribution matching, forward error correction, implementation, modulation, optical fiber communication, probabilistic shaping, reverse concatenation.

\section{INTRODUCTION}

Multilevel modulation formats have been intensively investigated in coherent optical communications due to the growing traffic demands and requirements for high spectral efficiency. To relax the signal-to-noise ratio (SNR) requirements for such formats, two independent approaches can be employed. The first is coding, or forward error correction (FEC), where hardand soft-decision schemes for fiber-optic communications have demonstrated bit error rates (BER) as low as $10^{-15}$ [1], [2]. The second is shaping, which aims at reducing the average signal energy by spherically confining the modulation levels in signal space.

Two shaping approaches can be distinguished; geometric shaping, which uses uniform (equiprobable) modulation levels that are selected to be more or less spherically distributed, and probabilistic shaping (PS), which is based on using low-amplitude constellation points more frequently. Both schemes aim at approximating a Gaussian distribution, which is capacity-achieving for the Gaussian channel. The ultimate SNR improvement for a multidimensional quadrature amplitude modulation (QAM) format relative to the Gaussian channel capacity approaches $\pi e / 6(1.53 \mathrm{~dB})$ as the number of dimensions tends to infinity, as shown, e.g., by Forney and Wei [3], who also demonstrated a simple approach to create multidimensional shaped constellations. Calderbank and Ozarow pointed out that an equiprobable (uniform) multidimensional constellation could be viewed as a lower-dimensional nonequiprobable (nonuniform) signaling scheme [4]. Kschischang and Pasupathy [5] studied nonuniform signaling (probabilistic shaping), and showed that high shaping gains could be realized already for relatively low dimensions and with limited complexity based on Huffman codes. However, the varying bit rate and synchronization problems of such schemes may preclude their usefulness in practical systems. In [6], these drawbacks were proposedly amended by keeping a constant bit-to-symbol rate at the expense of dropping (puncturing) bits.

As coherent optical communication assisted by digital signal processing has been realized [7], multidimensional signaling for optical links has regained interest, and efficient formats were proposed for the inherently four-dimensional optical signals [8]. For the nonlinear optic channel, variants of multidimensional geometric shaping [9]-[12] have been studied, as well as probabilistic shaping [13], [14], based on the scheme proposed in [6].

When combining coding and shaping, there are numerous issues to consider, e.g., the ordering of the schemes and the fact that the presence of one scheme may affect the performance of the other. While these issues were not discussed in the earlier works [3], [5], [8], [9], an outer FEC is often assumed [11], [12], or even necessary [6], [14].

On the other hand, recently Böcherer et al. [15] introduced probabilistic amplitude shaping (PAS) based on a reverse concatenation architecture, meaning that the FEC coding/decoding is performed inside the shaping algorithm, thus acting on nonuniformly distributed bits. This may realize improved performance and also enable rate adaptation in the shaping scheme rather the FEC, which may provide increased granularity. We note that reverse concatenation was indeed studied much earlier, in [16], and the use of soft-decision FEC was also investigated [17], [18]. Since its emergence, the PAS scheme in the transmitter and its termination in the receiver have been called distribution matching (DM) and distribution dematching (invDM), respectively, and we will use this terminology henceforth.

With the normal (nonreverse) concatenation architecture, invDM should be placed before soft-decision FEC decoding. Then soft demapping must convert the multidimensional fine-bit-resolution signals into logarithmic ratios of postprobabilities (a posteriori log-likelihood ratios or L-values) [19. Eq. (3.31)] for the decoder, which is prohibitively complex to implement for long codes. On the other hand, reverse con- 
catenation changes the invDM input/output signals to binary sequences. This makes the implementation of invDM much simpler, and enables the number of dimensions (the code length) to be larger, leading to better performance.

As the DM for reverse concatenation PS, constant composition DM (CCDM) based on arithmetic coding was proposed in [20] and examined for a fiber-optic channel in [21]. These state-of-the-art works show great performance by an almost ideal DM. However, there is room for simplification of these in high-throughput optical fiber communication systems. DM and invDM consume nonnegligible circuit resources, and in practice there will be implementation penalties caused by imperfect signal processing used in deployable hardware at high throughputs, relative to the achievable rate that assumes ideal DM and FEC. In addition, system performance monitoring must be reconsidered when we employ reverse concatenation PS; since the invDM may cause the BER to increase, postinvDM BER should be used instead of post-FEC BER.

In this paper, we propose and analyze in detail a DM scheme, which was briefly introduced in [22]. We compare it with CCDM. We also, for the first time, explain the shaped frame structure, show the result of the DM-to-invDM backto-back error insertion test, and suggest a method to estimate the post-invDM BER from the post-FEC BER.

This paper is organized as follows: In Secs. II and III, we describe the basic system model and relevant performance metrics. Implementation issues are discussed in Sec. IV] and the proposed DM and framing are discussed in Sec. V] Simulation results comparing our proposed scheme with CCDM are described in Sec. VI The proposed error-rate estimation is presented in Sec. VII A summary of the work and a future outlook are provided in Sec. VIII.

\section{SYSTEM MODEL}

Fig. 1 shows the system model and the corresponding performance metrics to be discussed later. We consider shaping of one-dimensional pulse amplitude modulation (PAM). However, its extension to two-dimensional QAM or optical fourdimensional modulation (two quadratures in two polarizations) is straightforward.

The notation is summarized in Tab. [. Throughout the paper, we use boldface symbols to denote vectors and calligraphic symbols to denote sets. At the transmitter side, the incoming binary sequence is framed into blocks of $\boldsymbol{A} \in \mathcal{B}^{N_{\text {block }}}$ bits by following, e.g., the modern optical transport network (OTN) standard protocol [23], [24] of $n \times 100 \mathrm{G}$ optical transport units (OTUCn). . In the DM, the binary sequence $\boldsymbol{A}^{\prime} \in \mathcal{B}^{N_{\mathrm{u}}}$ is converted to the shaped bit sequence $\boldsymbol{D} \in \mathcal{B}^{m N_{\mathrm{s}} / 2}$ including placeholders for FEC parity bits in the following block, where $m$ is the number of bits in a QAM symbol. The bit sequence $D$ determines the absolute amplitudes of $N_{\mathrm{s}}$ PAM symbols. A systematic binary FEC encoder generates $n_{\mathrm{c}}-k$ (uniformly distributed) parity bits from $k$ payload bits $\boldsymbol{D}^{\prime} \in \mathcal{B}^{k}$, and outputs the FEC codeword $\boldsymbol{B} \in \mathcal{B}^{n_{\mathrm{c}}}$. The bits $\boldsymbol{B}^{\prime} \in \mathcal{B}^{m}$ are mapped to the QAM symbol $\boldsymbol{X} \in \mathcal{X}^{2}$, where $\mathcal{X}$ denotes a PAM symbol set. A bullet in Fig. 1 indicates a length conversion, e.g., $\boldsymbol{B}$ and $\boldsymbol{B}^{\prime}$ have the same elements

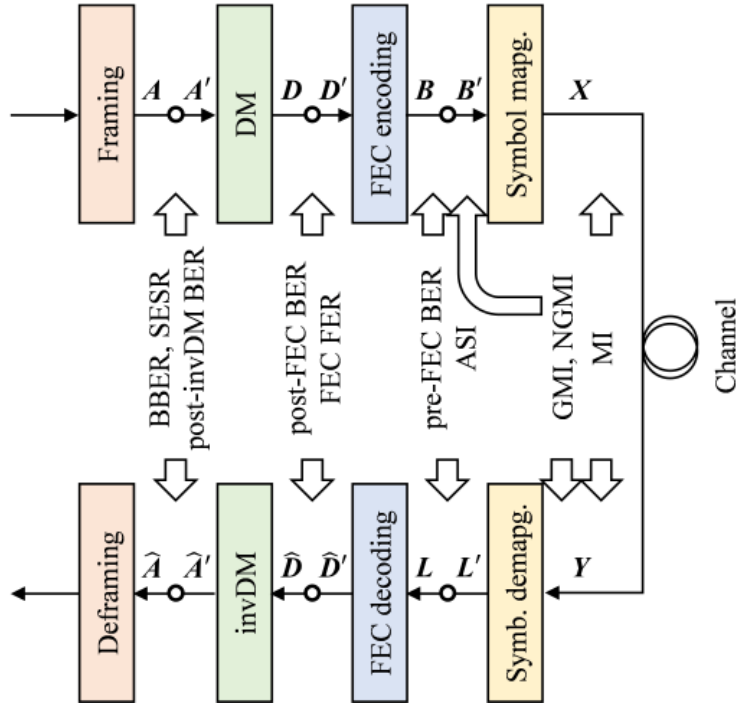

Fig. 1. System model including signal notation and performance metrics.

TABLE I

DEFINITIONS OF NOTATION.

\begin{tabular}{cl}
\hline Parameter & Description \\
\hline \hline$N_{\text {block }}$ & Block size of OTUCn, 130560n \\
$n$ & "n" in "OTUCn" \\
$N_{\mathrm{u}}$ & Number of DM input bits per DM word \\
$N_{\mathrm{s}}$ & DM word length (number of PAM symbols per DM word) \\
$n_{\mathrm{c}}$ & Number of information bits per FEC codeword \\
$k$ & Number of parity bits per FEC codeword \\
$m$ & Number of bits per QAM symbol \\
$\mathcal{B}$ & $\{0,1\}$ \\
$\mathcal{X}$ & $2^{m / 2}$-ary PAM symbol set for even $m(m \geq 2)$ \\
$\mathcal{R}$ & Real number set
\end{tabular}

from $\mathcal{B}$ but have different lengths of $n_{\mathrm{c}}$ and $m$ for interfacing, respectively, the FEC encoding output and symbol mapping input.

At the receiver side, the received QAM symbol $\boldsymbol{Y} \in \mathcal{R}^{2}$ is demapped by bit-metric decoding to a posteriori $\mathrm{L}$-values $\boldsymbol{L}^{\prime} \in \mathcal{R}^{m}$ [19. Eq. (3.31)], where $\mathcal{R}$ denotes a real number set. Based on the L-values $\boldsymbol{L} \in \mathcal{R}^{n_{\mathrm{c}}}$, FEC decoding recovers the payload bits $\widehat{D}^{\prime} \in \mathcal{B}^{k}$. The decoded bit sequence $\widehat{\boldsymbol{D}} \in$ $\mathcal{B}^{m N_{\mathrm{s}} / 2}$ is dematched to $\widehat{\boldsymbol{A}}^{\prime} \in \mathcal{B}^{N_{\mathrm{u}}}$, and finally $\widehat{\boldsymbol{A}} \in \mathcal{B}^{N_{\text {block }}}$ is deframed.

\section{Performance Metrics And Performance MONITORING}

\section{A. Achievable rates and metrics as post-FEC BER predictor}

Traditionally, the performance requirement for the optical layer has been a BER after FEC decoding down to $10^{-15}$ following standards for fiber communications [2], [25]. More modern performance metrics are based on information theory, which quantifies achievable information rates (AIRs) to lowerbound the capacity. The mutual information of the transmitted symbol $\boldsymbol{X}$ and the received symbol $\boldsymbol{Y}, I(\boldsymbol{X} ; \boldsymbol{Y})$, is such an AIR, which is achievable with nonbinary coding, bitinterleaved coded modulation (BICM) with iterative demapping, or multilevel coded modulation [26]. For BICM without iterative demodulation, an AIR is given by the generalized 
mutual information (GMI) [19, Eq. (4.55)]. The GMI works as a good post-FEC BER predictor for uniform and independent pragmatic signaling [27]. A practical, nonideal FEC has a rate loss, e.g., the required FEC code rate $R_{\mathrm{c}}$ for a post-FEC BER $<10^{-15}$ is smaller than the normalized GMI (NGMI) GMI $/ m$ [27, Tab. III].

For reverse concatenation PS with matched bit-metric decoding (bmd), an AIR in bit per channel use (bpcu) is [15], [28]

$$
R_{\mathrm{bmd}}^{\mathrm{ps}}=H(\boldsymbol{X})-\sum_{i=1}^{m} H\left(B_{i} \mid \boldsymbol{Y}\right),
$$

where $H(\cdot)$ and $H(\cdot \mid \cdot)$ denote entropy and conditional entropy, resp., and $B_{i}$ denotes a bit at bit level index $i$ before QAM symbol mapping. $R_{\mathrm{bmd}}^{\mathrm{ps}}$ takes the same value as a modified expression of GMI applicable to PAS defined in [29. Eq. (1)] with a matched auxiliary channel $q$, which was also shown in [30, Sec. III]. Eq. (1) quantifies the rate as a common expression for pragmatic BICM and reverse concatenation PS. While $R_{\mathrm{bmd}}^{\mathrm{ps}}$ is the rate with ideal FEC and ideal DM, the information rate in bpcu with a practical FEC (discussed in the previous paragraph) is defined as

$$
R=\frac{N_{\mathrm{u}}}{N_{\mathrm{s}} / 2} \leq H(\boldsymbol{X})-\left(1-R_{\mathrm{c}}\right) m,
$$

where the symbol entropy $H(\boldsymbol{X})$ is calculated from the probability mass function (PMF) of the transmitted QAM symbol $\boldsymbol{X}, P_{\boldsymbol{X}}(\boldsymbol{x}), \boldsymbol{x} \in \mathcal{X}^{2}$. Note that $H(\boldsymbol{X})$ is equivalent to $2 H(X)$ in the case of PAS, where $X$ denotes a PAM symbol in $\mathcal{X}$. Non-ideal DM has a rate loss, i.e., difference between the entropy and the information rate, shown in [15, Sec. V-B], [31, Eq. (4)], [32. Eq. (21)] for PAS, which can be expressed as

$$
R_{\text {loss }}=H(\boldsymbol{X})-\left(1-R_{\mathrm{c}}\right) m-\frac{N_{\mathrm{u}}}{N_{\mathrm{s}} / 2} \geq 0
$$

for reverse concatenation PS. The inequality is not restricted to PAS but more general. For certain (high performance) DMs [20], [31]-[35], the loss is mainly due to a finite (insufficient) length of the DM word.

As post-FEC BER predictors for PS systems using QAM, the NGMI was proposed in [29. Eq. (6)] as

$$
\mathrm{NGMI}=1-\frac{2 H(X)-\mathrm{GMI}}{m},
$$

which means GMI $/ m$ in a non-shaped case.

The asymmetric information (ASI) [30], [36, Eq. (11)]

$$
\begin{aligned}
\mathrm{ASI} & =1-h\left(L_{\mathrm{a}}|| L_{\mathrm{a}} \mid\right) \\
& =1-\frac{1}{m} \sum_{i=1}^{m} h\left(B_{i} \mid L_{i}\right)
\end{aligned}
$$

was also proposed for the same purpose, where $h(\cdot)$ denotes differential (continuous) entropy. We denote the symmetrized $a$ posteriori $\mathrm{L}$-value, or asymmetric $\mathrm{L}-$ value, with $L_{\mathrm{a}}=(-1)^{B}$. $L$, where $B \in \mathcal{B}$ is a sample of $\boldsymbol{B}$, and $L \in \mathcal{R}$ is a sample of $\boldsymbol{L}$ corresponding to the sampled bit $B$.

In practice, soft-demapping circuits operate with a finite bit resolution, which causes a minor performance loss. Here
GMI, NGMI, and ASI can account for that loss because GMI is based on both the received symbol $\boldsymbol{Y}$ and the auxiliary channel $q$ [27, Sec. III-C] and the ASI is based on the Lvalues just before the FEC decoder, while (1) does not account for it. The post-FEC BER can be estimated from the NGMI or the ASI. However, since the performance requirement for reverse concatenation PS is a post-invDM BER of $<10^{-15}$, one needs to account for a potential BER increase due to the invDM operation. We will discuss this further in Sec. VII

\section{B. Block performance monitoring}

In many modern communication systems, higher-layer protocols apply packet-oriented transmission, where the whole packet is discarded if any bit therein is received in error. BER metrics such as post-FEC BER or post-invDM BER might not be well suited to characterize the performance of such systems. Instead, systems using OTN framing are evaluated based on the background block error rate (BBER) and the severely errored second rate (SESR) [23], [24]. A severely errored second is a second with over $30 \%$ block errors. The BBER is equal to the number of erroneous blocks normalized by the total number of transmitted blocks , disregarding those appearing during a severely errored second (the block size of OTUCn is given in Sec. III). The SESR is the probability of a severely errored second, and used as a long-term performance monitoring metric. As BBER is a more basic performance metric, we will not consider SESR in this paper.

The BBER requirement depends on the link conditions, but a typical value is, e.g., around $10^{-7}$. For reverse concatenation PS, a large $N_{\mathrm{s}}$ can cause a long error burst from the residual error after FEC decoding, although a large $N_{\mathrm{s}}$ leads to smaller DM rate loss $R_{\text {loss. }}$. As the block performance monitoring is critical for system design, we will simulate and analyze its behavior in the next section. Note that higher layer packets have smaller or larger sizes compared with the block size of OTUCn, so both the post-invDM BER and the BBER must be considered. In addition, we will consider the FEC frame error rate (FER), which is the probability of an erroneous FEC frame, or codeword, after FEC decoding.

\section{IMPLEMENTATION ISSUES}

The state-of-the-art performance of PAS DM is provided by a coding scheme for $m$-out-of- $n$ codes [37] or CCDM [20]. With this technique, a rate loss $R_{\text {loss }}$ of almost zero is possible, provided that the DM word-length is large enough (typically $\left.N_{\mathrm{s}} \sim 10^{3}-10^{4}\right)$. However, this requires a prohibitively complex hardware implementation. Recently, several attempts have been made to find a better balance between performance and complexity [31]-[35], [38]-[44]. In [38], [39], fixedlength to fixed-length conversion was used, which is simple enough to implement, but the performance is limited. In [40], a fixed-length to variable-length conversion scheme with small address size look-up tables (LUT) was considered, showing a bounded conversion speed variation. However, this scheme can cause a large error burst after invDM if there is an error before the invDM. A small LUT for a fixed-length to variablelength conversion was combined with a periodical uniformalization process in [41] to act as a fixed-length to fixed-length 
TABLE II

BITS $\left(\boldsymbol{B}^{\prime}\right)$ TO SYMBOL'S ABSOLUTE AMPLITUDE $(|X|)$ CONVERSION FOR 32-PAM.

\begin{tabular}{|cc|cc|cc|cc|}
\hline $\boldsymbol{B}^{\prime}$ & $|X|$ & $\boldsymbol{B}^{\prime}$ & $|X|$ & $\boldsymbol{B}^{\prime}$ & $|X|$ & $\boldsymbol{B}^{\prime}$ & $|X|$ \\
\hline \hline 0000 & 1 & 0110 & 9 & 1100 & 17 & 1010 & 25 \\
0001 & 3 & 0111 & 11 & 1101 & 19 & 1011 & 27 \\
0011 & 5 & 0101 & 13 & 1111 & 21 & 1001 & 29 \\
0010 & 7 & 0100 & 15 & 1110 & 23 & 1000 & 31 \\
\hline
\end{tabular}

conversion. However, practical implementations of the fixedlength to variable-length conversion requires deeply sequential processing, and a large amount of parallelization (i.e., a large chip area) is required to realize the high throughput. In [42][44], separate DM is applied to each modulation bit level for efficient implementation, and bit-wise DM like [37], [39], [41] can be combined with it. Its performance mainly depends on the bit-wise DM, and it is also useful for approaching the water-filling capacity on transmission over parallel channels having different SNRs [43]-[45]. Other approaches to improve the performance with limited DM word length include partitioning and combining short constant-composition DM words [31], enumerative sphere shaping and its approximations [32][34], and shell mapping [35]. Their implementability on highthroughput signal processing still needs to be investigated.

An FEC coding scheme has another drawback due to the throughput increase from reverse concatenation PS. Consider two cases, nonshaped BICM and reverse concatenation PS, assuming the same client (pre-DM) bitrates, same FEC code rates $R_{\mathrm{c}}$, and symbol rates. To distinguish the two cases, we introduce $m_{\mathrm{u}}$ and $m_{\mathrm{s}}$ as the number of modulation bits $m$ for the nonshaped and shaped cases, resp. The shaped signal requires $m_{\mathrm{s}} / m_{\mathrm{u}}$ times larger FEC throughput than the nonshaped one, because the bit rate is increased by the DM. Then the FEC circuit size and the power consumption will be increased by the ratio $m_{\mathrm{s}} / m_{\mathrm{u}}$ for the shaped case. Note that there is an FEC code rate restriction of $R_{\mathrm{c}} \geq\left(m_{\mathrm{s}}-1\right) / m_{\mathrm{s}}$ in the case of PAS to assign the uniformly distributed FEC parity bits to sign-bit positions without degrading the PMF of $P_{|X|}(|x|)$. In a generalization of the PAS, the parity bits can be placed outside the sign-bit, which relaxes this constraint [39].

\section{Proposed hierarchical DM (HiDM)}

Here we propose , and describe for the first time in detail, a hierarchical DM (HiDM) with reasonable complexity, thus compatible with practical hardware limitations. It was briefly introduced in [22].

Our aim is not to generate an arbitrary distribution of the transmitted symbols, but to approximate the MaxwellBoltzmann (MB), or quantized Gaussian, distribution, which is the most commonly considered distribution in probabilistic shaping research. The MB distribution with parameter $\lambda$ is given by the PMF

$$
P_{X}^{\mathrm{MB}}(x)=\frac{\exp \left(-\lambda x^{2}\right)}{\sum_{\xi \in \mathcal{X}} \exp \left(-\lambda \xi^{2}\right)}
$$

for any $x \in \mathcal{X}$. For a given base constellation and average symbol energy, the MB distribution maximizes the entropy

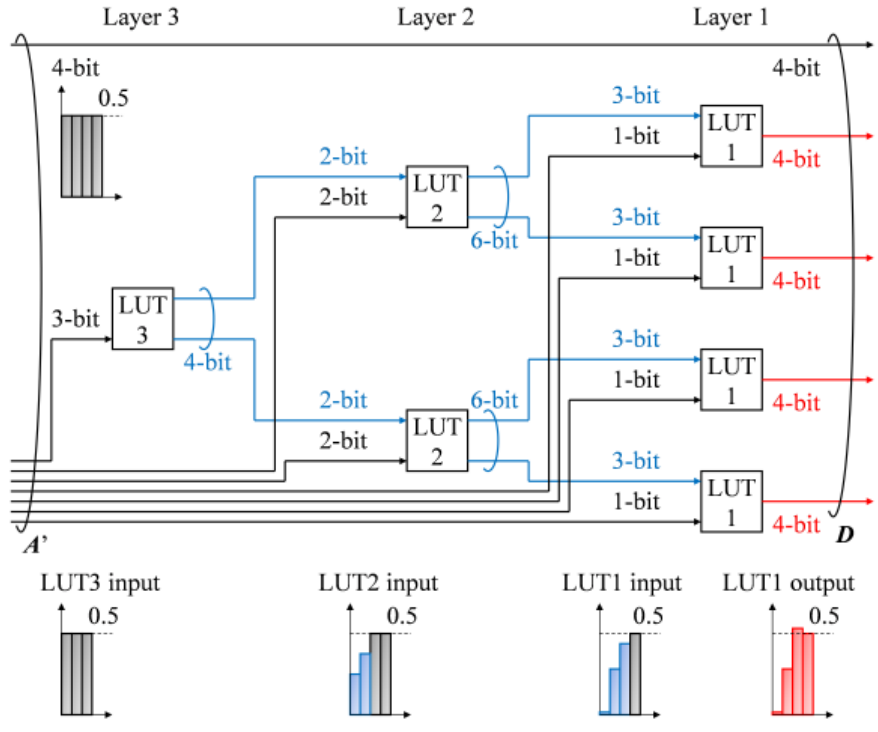

Fig. 2. Example of the proposed hierarchical DM (HiDM), where $N_{\mathrm{u}}=15$ uniform input bits $\boldsymbol{A}^{\prime}$ are converted into $m N_{\mathrm{s}}=20$ output bits $\boldsymbol{D}$ (4 uniform and 16 shaped bits). The non-shaped (uniform) bits will be used as sign bits of the 32-PAM symbols with $N_{\mathrm{s}}=4$. The bar charts illustrate probabilities of being ' 1 ' for the respective bits.

[5]. Furthermore, it approximates well the distribution that maximizes the mutual information over the Gaussian channel under an average energy constraint [6]. Conversely, if the information bit rate and base constellation are fixed, minimizing the average symbol energy results in a distribution similar to $\mathrm{MB}$, as will be exemplified in Sec. VI.

In principle, a DM with the best performance at a finite DM word length could be realized by a single look-up table (LUT), whose average symbol energy corresponding to the list of output bits $\boldsymbol{D}$ is minimized. However, when the DM word length $N_{\mathrm{s}}$ is large such as 20, the LUTs for DM and invDM are prohibitively large to be implemented in hardware. Thus we will demonstrate how to use small- to medium-size LUTs and combine them hierarchically to construct a larger set of output bit sequences than what each LUT can provide.

The input to the DM in Fig. 1 is the bit sequence $\boldsymbol{A}^{\prime} \in \mathcal{B}^{N_{\mathrm{u}}}$. Of those bits, $N_{\mathrm{u}}^{\mathrm{sb}}$ are converted by the shaping. The number of shaped bit levels per QAM symbol is $m^{\mathrm{sb}}$, which means that $m^{\mathrm{sb}} N_{\mathrm{s}} / 2$ of the $m N_{\mathrm{s}} / 2 \mathrm{DM}$ output bits $\boldsymbol{D}$ are shaped. The parameters $m, m^{\mathrm{sb}}, N_{\mathrm{u}}, N_{\mathrm{u}}^{\mathrm{sb}}, N_{\mathrm{s}}$ are dependent. The number of unshaped bits per DM word must be the number of FEC encoder input bits $R_{\mathrm{c}} m N_{\mathrm{s}} / 2$ subtracted by the number of shaped bits $m^{\mathrm{sb}} N_{\mathrm{s}} / 2$, i.e,

$$
N_{\mathrm{u}}-N_{\mathrm{u}}^{\mathrm{sb}}=\left(R_{\mathrm{c}} m-m^{\mathrm{sb}}\right) N_{\mathrm{s}} / 2 .
$$

Next we will explain how $N_{\mathrm{u}}^{\mathrm{sb}}$ uniform input bits can be converted into $m^{\mathrm{sb}} N_{\mathrm{s}} / 2$ nonuniform bits that generate probabilistically shaped output symbols.

\section{A. Principle of operation}

A small example of the proposed HiDM for generating a sequence of four PS-32-PAM symbols is shown in Fig. 2 . where $m / 2=5$, the shaped bit levels for a PAM symbol 
TABLE III

The CONTENTS OF THE LUTS SHOWN IN FIG. 2. INCLUDING EXPECTED SYMBOL ENERGY AND PMF.

\begin{tabular}{|c|c|c|c|c|c|c|c|c|c|c|c|}
\hline \multicolumn{4}{|c|}{ LUT3 } & \multicolumn{4}{|c|}{ LUT2 } & \multicolumn{4}{|c|}{ LUT1 } \\
\hline input & output & $\mathbb{E}\left[|X|^{2}\right]$ & PMF & input & output & $\mathbb{E}\left[|X|^{2}\right]$ & PMF & input & output & $|X|$ & PMF \\
\hline 000 & 0000 & 21 & $1 / 8$ & 0000 & 000000 & 5 & $1 / 8$ & 0000 & 0000 & 1 & $23 / 128$ \\
\hline 001 & 0001 & 41 & $1 / 8$ & 0001 & 000001 & 21 & $1 / 8$ & 0001 & 0001 & 3 & $23 / 128$ \\
\hline 010 & 0100 & 41 & $1 / 8$ & 0010 & 001000 & 21 & $1 / 8$ & 0010 & 0011 & 5 & $11 / 64$ \\
\hline 011 & 0101 & 61 & $1 / 8$ & 0011 & 001001 & 37 & $1 / 8$ & 0011 & 0010 & 7 & $11 / 64$ \\
\hline 100 & 0010 & 63 & $1 / 8$ & 0100 & 000010 & 53 & $1 / 16$ & 0100 & 0110 & 9 & $3 / 32$ \\
\hline 101 & 1000 & 63 & $1 / 8$ & 0101 & 010000 & 53 & $1 / 16$ & 0101 & 0111 & 11 & $3 / 32$ \\
\hline 110 & 0011 & 83 & $1 / 8$ & 0110 & 001010 & 69 & $1 / 16$ & 0110 & 0101 & 13 & $3 / 64$ \\
\hline 111 & 1100 & 83 & $1 / 8$ & 0111 & 010001 & 69 & $1 / 16$ & 0111 & 0100 & 15 & $3 / 64$ \\
\hline & & & & 1000 & 000011 & 101 & $1 / 32$ & 1000 & 1100 & 17 & $1 / 128$ \\
\hline & & & & 1001 & 011000 & 101 & $1 / 32$ & 1001 & 1101 & 19 & $1 / 128$ \\
\hline & & & & 1010 & 010010 & 101 & $1 / 32$ & 1010 & 1111 & 21 & 0 \\
\hline & & & & 1011 & 001011 & 117 & $1 / 32$ & 1011 & 1110 & 23 & 0 \\
\hline & & & & 1100 & 011001 & 117 & $1 / 32$ & 1100 & 1010 & 25 & 0 \\
\hline & & & & 1101 & 010011 & 149 & $1 / 32$ & 1101 & 1011 & 27 & 0 \\
\hline & & & & 1110 & 011010 & 149 & $1 / 32$ & 1110 & 1001 & 29 & 0 \\
\hline & & & & 1111 & 000100 & 165 & $1 / 32$ & 1111 & 1000 & 31 & 0 \\
\hline
\end{tabular}

$\boldsymbol{i}_{\mathrm{sb}}=(2,3,4,5), m^{\mathrm{sb}}=2\left|\boldsymbol{i}_{\mathrm{sb}}\right|=8, N_{\mathrm{u}}^{\mathrm{sb}}=11$, and $N_{\mathrm{s}}=4$ (i.e., four PAM symbols or two QAM symbols). For simplicity, we assume here an FEC code rate of $R_{\mathrm{c}}=1$. Hence, the DM has 15 uniform input bits and 20 output bits. Four of the input bits remain untouched by the DM, while 11 input bits are converted into 16 shaped bits. Tab. III shows the assumed bit-to-symbol mapping table for 32-PAM. The DM consists of three small LUTs, called LUT1, LUT2, and LUT3, hierarchically organized in a tree-like structure with three layers. Each layer comprises one or more LUTs, which are the same within each layer. The LUT contents including the expected one-dimensional energy $\mathbb{E}\left[|X|^{2}\right]$ and probabilities of each small-DM word, PMF, are shown in Tab. III

At first, three uniformly distributed information bits are input to LUT3 and converted into four output bits, which will act as constraint bits in the connected lower-layer DMs. The four constraint bits are separated into two lines of two constraint bits, each having mark ratios ${ }^{1}$ of 0.25 and 0.375 , which together with two uniformly distributed information bits are fed into each LUT2. The behavior of the resulting bit probability statistics, i.e., mark ratios, are shown by the bar charts in Fig. 2. The constraint bits are chosen so that the expected symbol energy becomes small. LUT2 converts four input bits into six output bits, which are again separated into two lines and treated as constraints bits in the next layer. Inputting each set of three constraint bits having mark ratios of $0.016,0.281$, and 0.438 with one uniformly distributed information bit, each LUT1 generates four output bits having mark ratios of $0.016,0.300,0.531$, and 0.5 shown by red bar charts. Note that the output bits from the LUTs are dependent. The output bits from LUT1 will now determine the absolute amplitudes $|\boldsymbol{X}|$ of the symbol sequence $\boldsymbol{X}$. In this example, the PMF of $|X|$ becomes nonuniform and one-sided MB-like, as shown in the last column of Tab. III. The generated DM words are not constant composition.

The expected energy in Tab. III is derived from the expected

\footnotetext{
${ }^{1} \mathrm{~A}$ "mark" is a bit equal to ' 1 ', and the probability for that bit being ' 1 ' is commonly referred to as "mark ratio".
}

energies in the lower layers. E.g., the LUT2 entry with output bits '000 001' generates input sequences ' $000 u$ ' and '001u' to layer 1 , where ' $u$ ' denotes a uniform choice of ' 0 ' or ' 1 '. The average energies of these sequences are $\left(1^{2}+3^{2}\right) / 2=5$ and $\left(5^{2}+7^{2}\right) / 2=37$, resp., and hence $\mathbb{E}\left[|X|^{2}\right]=(5+37) / 2=21$ for '000 001' in LUT2, as shown on the second row of Tab. III Each list is sorted from lower to higher energy, and lower chosen more frequently by the constraints from higher layers.

From these values, the information rate of the PAM symbol is $15 / 20 \times 5=3.75$, and the entropy is 3.93 , and the rate loss $R_{\text {loss }}$ becomes 0.18 bits per dimension. When the five output bits are mapped to a Gray-coded 32-PAM symbol, the expected energy per one-dimensional symbol is 57 . The constellation gain, defined as [5, Eq. (8)]

$$
G=\frac{d_{\min }^{2}\left(2^{\beta}-1\right)}{6 E},
$$

where $d_{\min }, \beta$, and $E$ denote minimum Euclidean distance, spectral efficiency (bpcu) at an FEC code rate of 1, and average symbol energy, resp., will be $0.22 \mathrm{~dB}$.

Additional flexibility and finer granularity can be obtained by increasing the number of layers, the number of LUTs connected between layers, the number of input information bits to each layer's LUT, and the number of constraint bits in each layer, which gives better adaptation of the PMF $P_{X}(x)$ to an approximated MB distribution and more significant performance improvements. Each LUT and thus the whole DM function are one-to-one mappings, so that they are reversible for the invDM. All constraint bits are used for the selection of symbols to minimize the expected symbol energy. The recipes for designing the LUTs will be discussed next in Sec. V-B.

\section{B. Recipe for LUT-tree construction and LUT content design}

In this section, we describe how to design the HiDM, to realize flexible rates and/or maximization of the constellation gain at given input/output rates. We denote by $L$ the number of layers and by $T_{\ell}$ the number of LUTs in layer $\ell=1, \ldots, L$, where $T_{L}=1$. The number of input bits to each LUT on layer 
$\ell$ is denoted by $v_{\ell}=r_{\ell}+s_{\ell}$, where $r_{\ell}$ bits come from an LUT on layer $\ell+1$ and $s_{\ell}$ bits are take directly from the DM input. Each LUT on layer $\ell+1$ is connected to $t_{\ell}=T_{\ell} / T_{\ell+1}$ different LUTs on layer $\ell$, and hence the number of LUT output bits is $u_{\ell+1}=t_{\ell} r_{\ell}$. The total number of shaped DM output bits is $T_{1} u_{1}$, corresponding to $T_{1} u_{1} /(m / 2-1)$ PAM symbols, and the corresponding number of DM input bits is $N_{\mathrm{u}}^{\mathrm{sb}}=\sum_{\ell} T_{\ell} s_{\ell}$. The number of unshaped bits passing untouched through the HiDM is given by (8).

Using this notation, the HiDM LUT tree is designed by repeating the following steps for $\ell=1,2, \ldots$ :

1) Select the number of output bits $u_{\ell}$ of each LUT in layer $\ell$. The number of output bits should not be too large, like 20 or above, since it would require a big memory size in the invDM. Suitable values are in the range from 2 to 14 bits, e.g., $\left(u_{1}, u_{2}, u_{3}\right)=(4,6,4)$ bits in Fig. 2

2) Select the number of input bits $s_{\ell}$ that are taken from the DM input, observing that the rate will not be so flexible if $s_{\ell} / u_{\ell}$ is small. Also select the number of input bits $r_{\ell}$ that come from LUTs in layer $\ell+1$. The higher-layer LUT storage size will be big if this value is too high. In Fig. 2. $\left(s_{1}, s_{2}, s_{3}\right)=(1,2,3)$ and $\left(r_{1}, r_{2}, r_{3}\right)=(3,2,0)$.

3) Select the number of LUTs $t_{\ell}$ in layer $\ell$ connected to a single LUT in layer $\ell+1$.

4) Terminate when number of output PAM symbols $T_{1} u_{1} /(m / 2-1)$ is sufficient, where $T_{1}=t_{1} t_{2} \cdots t_{\ell}$. Let $L=\ell$.

Once the LUT-tree configuration is established, the contents in each LUT is determined by the following sorting method. The contents of the LUTs are another critical matter for HiDM, so its basic concept is shown here, again using the small example shown in Tab. III

1) List the output DM words of each LUT in layer 1 by sorting them after increasing symbol energy, as shown in LUT1 in Tab. III There are $2^{u_{1}}$ candidates, and the top $2^{v_{1}}$ words (that is, those having smallest symbol energy) are selected.

2) For $\ell=2, \ldots, L$, calculate the expected energies for all possible output words from the LUT in layer $\ell$, using the procedure described in Sec. V-A Sort these words by increasing energy, as shown in LUT2 in Tab. IIII There are $2^{u_{\ell}}$ candidate words, of which the top $2^{v_{\ell}}$ are valid.

If we change the parameters $\left(r_{1}, s_{1}, u_{2}, s_{3}\right)$ from $(3,1,6,3)$ in Fig. 2 into $(2,2,4,4)$, the number of input bits and the DM information rate in the HiDM becomes 20 and 20/20 = 1 , resp. We can also change $s_{\ell}$ to have a desired DM code rate with a granurality of $1 / 20$. When implementing a fully flexible-rate HiDM in hardware, the LUT architecture should support a DM information rate of 1 , an external circuit should control the used/unused information bit input path, and the LUT contents should ignore the unused bits.

\section{Complexity}

The complexity of the required circuitry is dominated by the number of stored bits in the LUTs, which determines the area in the hardware implementation. The number of stored bits for all LUTs in layer $\ell$ is $T_{\ell} 2^{v_{\ell}} u_{\ell}$. In the example of Fig. 2, the number of stored bits are 32, 192, and 256 in layers 3, 2, and 1 , resp., thus in total 480 . The corresponding LUTs on the invDM side have $T_{\ell} 2^{u_{\ell}} v_{\ell}$ stored bits, which in the example gives $48+512+256=816$ bits in total for the three layers. If we would instead use a single LUT, i.e., 11-bit input and 16-bit output on the DM side and conversely on the invDM side, the number of stored bits would be $2^{11} \times 16=32768$ and $2^{16} \times$ $11=720896$, resp. Thus, HiDM saves circuit resources by a factor of 68 and 883 in the DM and invDM, resp., already in this small-scale example. Note that the performance of HiDM is always inferior to a DM with a single LUT that can have the optimum list (lowest average symbol energy) for the DM words, but the gain in complexity and memory size can be considerable,

HiDM with DM word length $N_{\mathrm{s}} \approx 100$ or 1000 can be realized by LUTs with reasonable sizes. The DM does thus not require complex operations such as integer additions or multiplications. The conversion process is purely fixed-length input and fixed-length output (there is no variable length part). Either bit-wise [42] or symbol-wise [20] DMs are possible, and MBlike distributions can be approximated with high granularity. This DM achieves a high throughput because the architecture consists of a fully parallelized input/output configuration, as well as a bit-scramble selector and a permutation mapper [39]. The hierarchical operation is fully pipelined, so a small number of instances is enough.

In contrast, arithmetic coding based DM, e.g., a coding scheme for $m$-out-of- $n$ codes [37] and CCDM [20] requires high-precision integer multiplications, which limits the throughput, and a large number of instances (at least $\sim N_{\mathrm{s}}$ ) would be require $2^{2}$. Then the equivalent DM word-length is in the order of $N_{\mathrm{s}}^{2}$ or more, as will be further discussed in the next section.

\section{Frame structure}

An example of a frame structure used for the transmission line (channel) with the CCDM (a) and the proposed HiDM (b) are shown in Fig. 3 . The blue (filled) rectangles show one DM word. As discussed in Footnote 2, while one CCDM circuit generates one symbol per clock-cycle due to the sequential operation, HiDM generates many symbols per clock-cycle because of the parallel output interface. This leads to the difference in Figs. 3 a) and (b) The FEC is assumed to be a DVB-S2 low-density parity-check code [46] with codeword length $n_{\mathrm{c}}=64800$ and code rate $R_{\mathrm{c}}=5 / 6$. The modulation

\footnotetext{
${ }^{2}$ Here "instance" refers to the number of DM circuits that have to be implemented in parallel on the ASIC. While the latest CMOS logic circuitry can operate at hundreds of $\mathrm{GHz}$, the throughput of optical communication links reaches hundreds of $\mathrm{Gb} / \mathrm{s}$ to $\mathrm{Tb} / \mathrm{s}$. CCDM requires sequential operation, so one CCDM circuit can generate the absolute amplitude of one PAM symbol per clock cycle using a straight-forward implementation, although it depends on the logical circuit design. Then to generate $N_{\mathrm{S}}$ PAM absolute amplitudes, $N_{\mathrm{s}}$ clock-cycles are needed. Until completing one DM word, one cannot input information bits for the next DM word. Thus $N_{\mathrm{s}}$ parallel CCDM circuits are necessary for real-time operation. When the clock speed is $500 \mathrm{MHz}, 320$ Gsymbol/s is achieved, which would be sufficient for the required throughput. On the contrary, HiDM outputs, e.g., $320 \mathrm{Gsymbol} / \mathrm{s}$ per HiDM circuit at a clock-cycle, so it requires just a few instances.

${ }^{3}$ A HiDM-like frame structure for CCDM can be realized by rearrangement of the memory, but it will significantly increase the latency.
} 


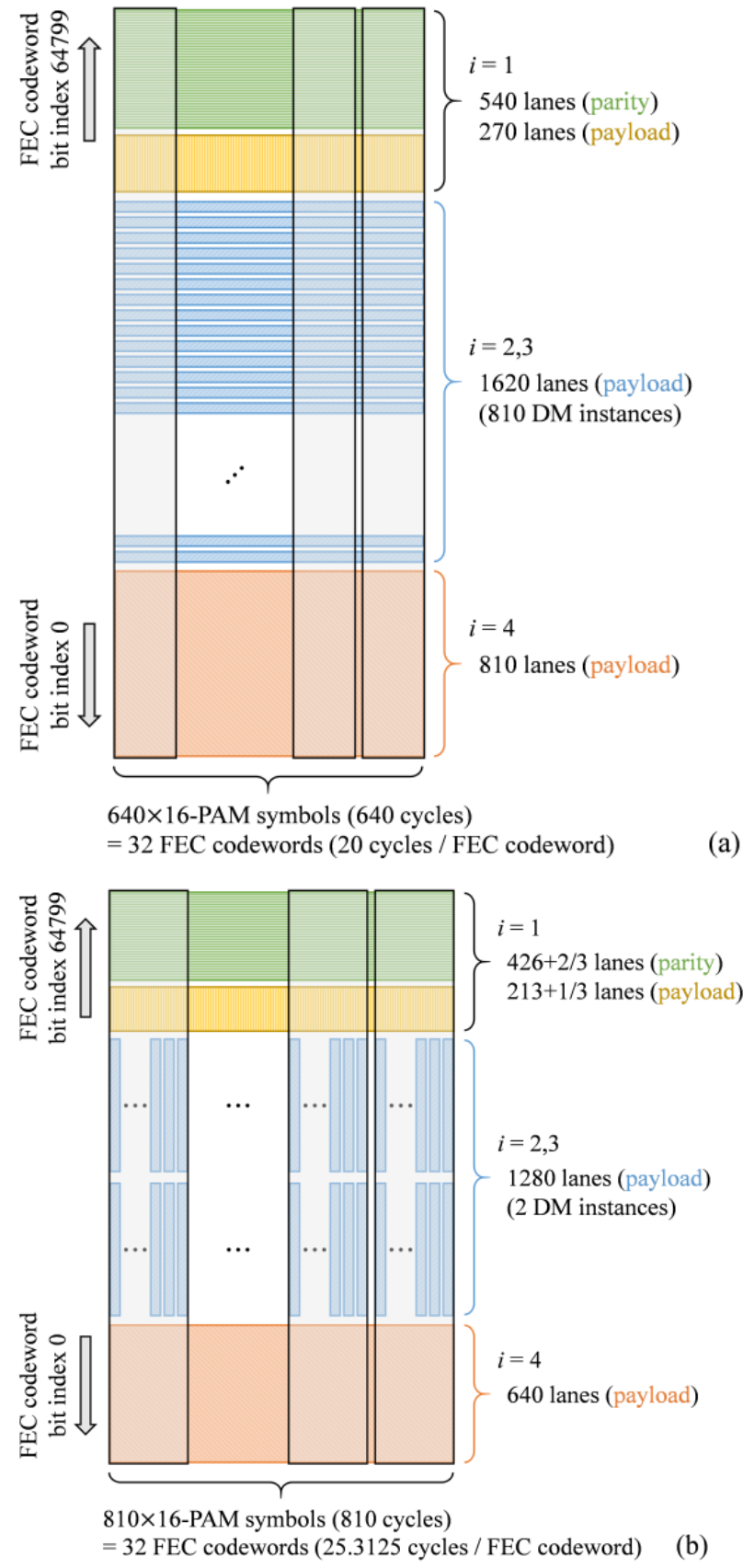

Fig. 3. Frame structure with (a) CCDM or with (b) HiDM. One blue (filled) rectangle shows one DM word.

format is Gray-coded 16-PAM. The most significant $(i=1$, sign-bit) and the least significant $(i=4)$ bit-levels for the 16PAM symbols are not shaped to simplify the implementation. Thus, $m^{\mathrm{sb}} / 2=2$ and the shaped bit levels are $\boldsymbol{i}_{\mathrm{sb}}=\{2,3\}$. The number of input bits $N_{\mathrm{u}}^{\mathrm{sb}}$ is 1014 or 507, the number of output PAM symbols $N_{\mathrm{s}}$ is 640 or 320 , and the number of output bits $m^{\mathrm{sb}} N_{\mathrm{s}} / 2$ is 1280 or 640 for CCDM or HiDM, resp., in this example. The HiDM is designed using seven layers. In layer 1, there are 64 small LUTs, each having $u_{1}=10$ output bits. Thus, the total number output bits from the proposed DM is 640 , which equals $m^{\mathrm{sb}} N_{\mathrm{s}} / 2$. The required storage sizes for HiDM with these parameters and two instances are manageable, with values of $3.7 \times 10^{6}$ for DM and $6.8 \times 10^{6}$ for invDM, while that for two instances using a single LUT would be prohibitively large, i.e., $2 \cdot 2^{507} \cdot 640=5.4 \times 10^{155}$ for $\mathrm{DM}$ and $2 \cdot 2^{640} \cdot 507=4.6 \times 10^{195}$ for invDM. Thus, HiDM reduces the total storage size by more than $10^{194}$ times, though there is a performance drawback compared to using a single LUT.

The information rate $R$ is $5.83 \mathrm{bpcu}$, which is equivalent to 128 -QAM with $R_{\mathrm{c}}=5 / 6$. The equivalent word-length for CCDM and HiDM are $518400\left(>N_{\mathrm{s}}^{2}\right)$ and 320, resp. While $810 \mathrm{DM}$ words are equally mapped to $32 \mathrm{FEC}$ codewords in parallel for the CCDM, 50 or $51 \mathrm{DM}$ words are included in an FEC codeword sequentially for the HiDM. The bit indices 0 53999 of the FEC codeword correspond to the payload bits and indices 54000-64799 to the parity bits. The less significant bits are placed at lower bit indices of the FEC codeword to balance the pre-FEC performance and the FEC decoding capability.

\section{Simulations}

To evaluate the post-FEC and post-invDM performance with reverse concatenation PS, we conducted numerical simulations of PS-256-QAM transmission over the Gaussian channel. The same combinations of FEC and PS were examined as in Sec. V] i.e., the DVB-S2 low-density parity-check code with $R_{\mathrm{c}}=5 / 6$. The number of decoding iterations was 20 and more than 1600 FEC codewords were examined per simulation. The soft-demapping input and output interfaces were quantized with 7 and 4 bits, resp., which gives less than $0.1 \mathrm{~dB}$ SNR penalty. Two DM schemes were implemented, HiDM and CCDM. For comparison we also simulated nonshaped BICM 128-QAM with a Gray-like labeling [47, Slide 10] $]^{4}$ Its most significant bit $(i=1)$ and parts of the second significant bit ( $i=2$, which are sign bits) were occupied by the FEC parity bits, for the same information rate 5

Before evaluating the error rates, we summarize the statistics of the PMF $P_{|X|}(\cdot)$ in Tab. IV for CCDM, HiDM, and the MB distribution with $\lambda=0.1373$. We also give the average symbol energy $E$, the two-dimensional entropy $2 H(X)$, the spectral efficiency $\beta$ at $R_{\mathrm{c}}=1$, the DM rate loss $R_{\text {loss }}$ (which equals $2 H(X)-\beta$ in this case), and the constellation gain $G$. For CCDM, $\beta$ can be increased to $2 \cdot(2+1015 / 640)$ at a target PMF of (7) with $\lambda=0.0133$, but it was reduced to $2 \cdot(2+1014 / 640)$ to fit to the frame. Its influence on $R_{\text {loss }}$ and $G$ is 0.004 bpcu and $0.01 \mathrm{~dB}$, resp. HiDM shows $0.13 \mathrm{~dB}$ and $0.39 \mathrm{~dB}$ performance loss compared with CCDM and the MB distribution, resp. If the DM word length $N_{\mathrm{s}}$ of CCDM is decreased from 640 to 320 , then the constellation gain $G$ becomes almost the same as HiDM.

Fig. 4 shows the simulation results in terms of post-FEC BER, post-invDM BER, FER, and BBER as a function of the SNR. Here we assume the use of OTUC1 framing, so the

\footnotetext{
${ }^{4}$ There is no perfect Gray code for cross-QAM constellations such as this 128-QAM, which leads a performance drawback.

${ }^{5}$ Performance comparisions between shaped and non-shaped signals raises the question of whether to use different base constellations or different FEC code rates. For ease of hardware implementation and flexible setting of spectral efficiencies, we chose the same FEC code rate but different base constellations in this paper.
} 
TABLE IV

RESULTING STATISTICS BY SHAPING.

\begin{tabular}{|c|cc|c|c|}
\hline & \multicolumn{2}{|c|}{ CCDM } & HiDM & MB \\
\hline \hline$N_{\mathrm{s}}$ (PAM-symbol) & 640 & 320 & 320 & - \\
$P_{|X|}(1)$ & 0.2484 & 0.2453 & 0.2376 & 0.2628 \\
$P_{|X|}(3)$ & 0.2484 & 0.2453 & 0.2376 & 0.2355 \\
$P_{|X|}(5)$ & 0.1625 & 0.1625 & 0.1684 & 0.1891 \\
$P_{|X|}(7)$ & 0.1625 & 0.1625 & 0.1684 & 0.1360 \\
$P_{|X|}(9)$ & 0.0695 & 0.0719 & 0.0757 & 0.0877 \\
$P_{|X|}(11)$ & 0.0695 & 0.0719 & 0.0757 & 0.0506 \\
$P_{|X|}(13)$ & 0.0195 & 0.0203 & 0.0183 & 0.0262 \\
$P_{|X|}(15)$ & 0.0195 & 0.0203 & 0.0183 & 0.0121 \\
\hline$E$ & 72.50 & 74.00 & 74.70 & 68.31 \\
$2 H(X)(\mathrm{bpcu})$ & 7.214 & 7.242 & 7.252 & 7.169 \\
$\beta$ at $R_{\mathrm{c}}=1(\mathrm{bpcu})$ & 7.169 & 7.169 & 7.169 & 7.169 \\
$R_{\text {loss }}$ (bpcu) & 0.045 & 0.073 & 0.083 & 0 \\
$G(\mathrm{~dB})$ & 1.186 & 1.097 & 1.056 & 1.444 \\
\hline
\end{tabular}

block size is 130560 bits. The post-FEC BER at low BERs is similar among the bit levels, due to the bit level mapping in the FEC codeword space [15]. CCDM shows $0.13 \mathrm{~dB}$ lower required SNR than HiDM at a post-FEC BER of $10^{-6}$, and 128-QAM BICM performed further $0.8 \mathrm{~dB}$ worse than HiDM. The difference in the required SNR of $0.13 \mathrm{~dB}$ between CCDM and HiDM agrees well with the difference in constellation gain $G$ shown in Tab. IV] If we decrease the DM word length $N_{\text {s }}$ to 320 for CCDM, the required SNR is expected to increase by around $0.1 \mathrm{~dB}$, according to the difference in constellation gain $G$.

The error rate increase by the invDM for the reverse concatenation PS is characterized by the post-invDM BER $\mathcal{E}_{\text {post-iDM }}$ to post-FEC BER $\mathcal{E}_{\text {post-FEC }}$ ratio,

$$
r_{\mathcal{E} 1}=\frac{\mathcal{E}_{\text {post-iDM }}}{\mathcal{E}_{\text {post-FEC }}}
$$

which are 200 and 5.5 for CCDM and HiDM, resp., at around $\mathcal{E}_{\text {post-FEC }}=10^{-6}$. This relation is expected to be valid also at lower error rates, according to the analysis in the next section.

To quantify the amount of burst errors after the invDM, the ratio of BBER $\mathcal{E}_{\text {block }}$ to post-invDM BER $\mathcal{E}_{\text {post-iDM, }}$,

$$
r_{\mathcal{E} 2}=\left\{\begin{array}{lc}
\mathcal{E}_{\text {block }} / \mathcal{E}_{\text {post-iDM }} & (\mathrm{CCDM}, \mathrm{HiDM}) \\
\mathcal{E}_{\text {block }} / \mathcal{E}_{\text {post-FEC }} & (\mathrm{BICM})
\end{array}\right.
$$

is useful, which is found to be 500,11000 , and 20000 for CCDM-based PS-256-QAM, HiDM-based PS-256-QAM, and BICM 128-QAM, respectively, as shown in Fig. 4 If $r_{\mathcal{E} 2}$ is smaller, the erroneous frame has a larger number of errors, so the error bursts are more severe. The error burstiness of HiDMbased PS-256-QAM is $11000 / 500=22$ times lower than with CCDM. It means that the HiDM can be concatenated with an outer hard-decison FEC if needed because of the significant reduction of the burstiness of the residual errors.

\section{POST-INVDM PERFORMANCE ESTIMATION AT LOW ERROR RATES}

We estimated the post-invDM BER from the post-FEC BER by inserting random errors into each DM word before the invDM operation. Fig. 5 shows the simulation configuration for the DM to invDM back-to-back error insertion test. As
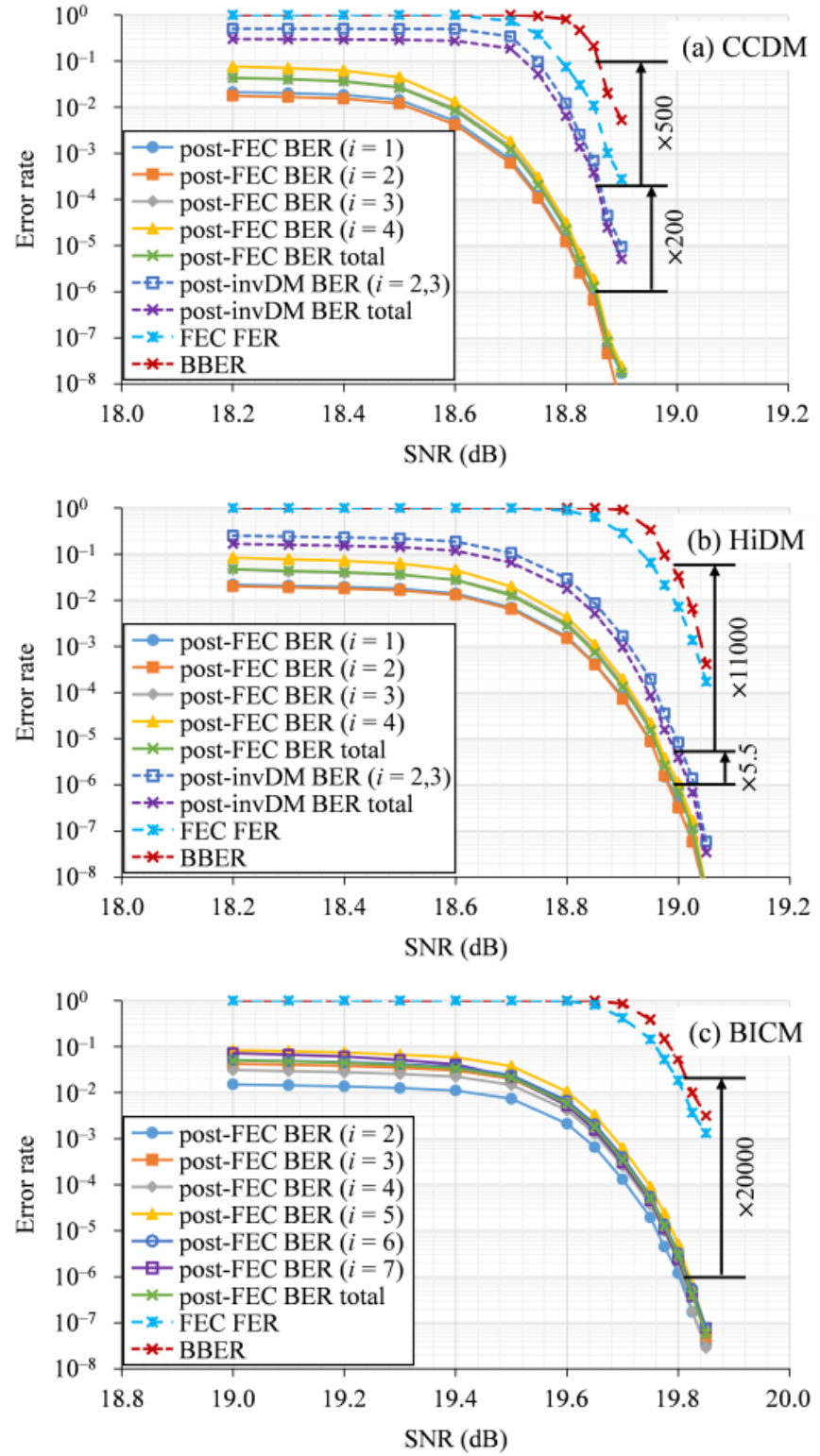

Fig. 4. Simulation results of (a) CCDM-based PS-256-QAM, (b) HiDM-based PS-256-QAM, and (c) BICM-based 128-QAM.

before, the shaped bit levels are $\boldsymbol{i}_{\mathrm{sb}}=\{2,3\}$ and $m^{\mathrm{sb}} / 2=$ 2. The number of information bits $N_{\mathrm{u}}^{\mathrm{sb}}=1014$ and 507 for CCDM and HiDM, resp. The information bits were fed to the DM, $m^{\text {sb }} N_{\mathrm{s}} / 2=2 N_{\mathrm{s}}$ shaped bits were generated, errors were inserted into the shaped bits, and the invDM recovered the shaped information.

Fig. 6 shows the simulated post-invDM BER $\mathcal{E}_{\text {post-iDM }}^{\text {sb }}$ as a function of the number of erroneous bits per DM word, both computed for the shaped bits only. The expected value of $\mathcal{E}_{\text {post-iDM }}^{\mathrm{sb}}$ for CCDM is 0.5 for any number of errors, whereas for HiDM it increases from 0.027 for a single error to 0.16 for 10 errors. The expected number of errors after post-invDM $\alpha$ is 507 for CCDM and 13.4 for HiDM with a single bit error.

The ratio $r_{\mathcal{E} 1}$ of 200 or 5.5 in the previous section corresponds to the expected error count under the single-error input assumption. Although the FEC decoder can output an error 


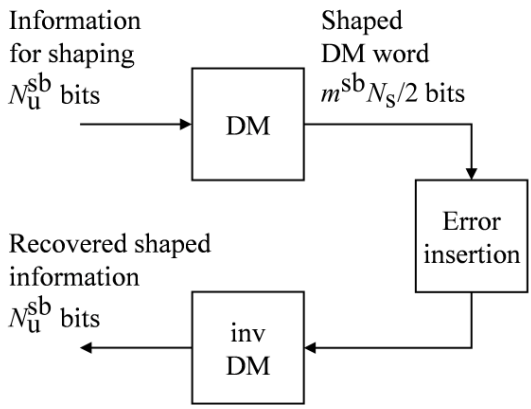

Fig. 5. Simulation setup for DM to invDM back-to-back error insertion test.

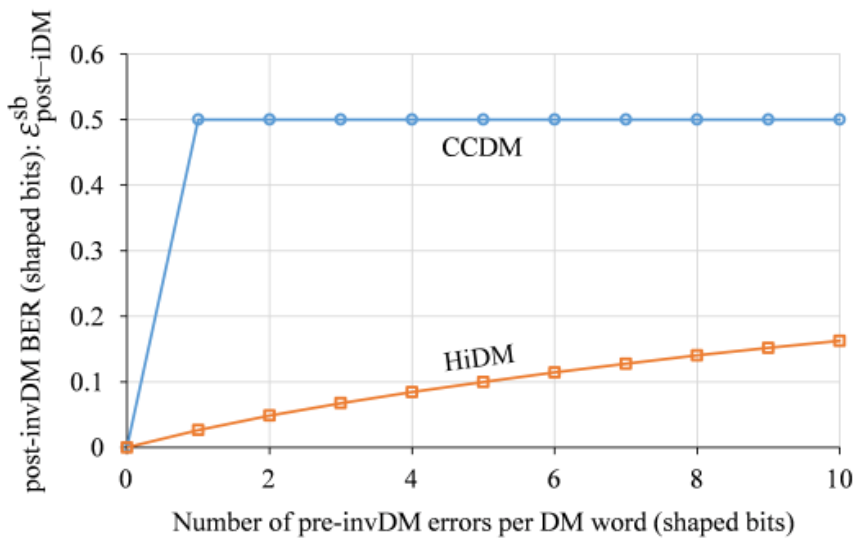

Fig. 6. Simulated post-invDM BER under a single error per DM word at the input of the invDM for PS-256-QAM.

burst, at least the bit-level dependence is weak according to the simulation results in Fig. 4. Under the single-error input condition, the post-invDM BERs for the shaped bits and the total bits, $\mathcal{E}_{\text {post-iDM }}^{\text {sb }}$ and $\mathcal{E}_{\text {post-iDM }}^{\text {total }}$, are bounded as

$$
\begin{aligned}
& \mathcal{E}_{\text {post-iDM }}^{\text {sb }} \leq \min \left\{\frac{\alpha \cdot m^{\mathrm{sb}} N_{\mathrm{s}} / 2}{N_{\mathrm{u}}^{\mathrm{sb}}} \mathcal{E}_{\text {post-FEC }}, \frac{1}{2}\right\}, \\
& \mathcal{E}_{\text {post-iDM }}^{\text {total }} \leq \gamma_{\text {in }} \mathcal{E}_{\text {post-iDM }}^{\mathrm{sb}}+\left(1-\gamma_{\text {in }}\right) \mathcal{E}_{\text {post-FEC }} .
\end{aligned}
$$

The fractions of shaped DM input and output bits (i.e., the fractions of $\boldsymbol{A}^{\prime}$ and $\boldsymbol{D}$ that are shaped in the DM) are, resp.,

$$
\begin{aligned}
\gamma_{\text {in }} & =\frac{N_{\mathrm{u}}^{\mathrm{sb}}}{N_{\mathrm{u}}}, \\
\gamma_{\text {out }} & =\frac{m^{\mathrm{sb}}}{R_{\mathrm{c}} m} .
\end{aligned}
$$

The BBER can be bounded as

$$
\mathcal{E}_{\text {block }} \leq \min \left\{\frac{N_{\text {block }}\left(\gamma_{\text {out }} \theta+1-\gamma_{\text {out }}\right)}{k \cdot N_{\mathrm{u}} /\left(R_{\mathrm{c}} m N_{\mathrm{s}} / 2\right)} \mathcal{E}_{\mathrm{FEC}-\mathrm{fr}}, 1\right\},
$$

where the FEC FER $\mathcal{E}_{\text {FEC-fr }}$ is also bounded as

$$
\mathcal{E}_{\text {FEC-fr }} \leq \min \left\{k \cdot \mathcal{E}_{\text {post-FEC }}, 1\right\} .
$$

The parameters $\alpha$ and $\theta$, which denote the number of output error bits of the invDM with a single-error input and the number of OTUC1 block errors with a single FEC codeword error, resp., are shown in Tab. $\mathrm{V}$, along with $\gamma_{\text {out }}$ and $\gamma_{\text {in. In }}$. the simulations, the number of information bits for the FEC is $k=54000$. If the expected number of errors in an erroneous
TABLE V

SINGLE-ERROR PARAMETERS

\begin{tabular}{cccc}
\hline \hline & CCDM & HiDM & BICM \\
\hline$\alpha$ & 507 & 13.4 & - \\
$\theta$ & 32 & $\leq 2$ & 1 \\
$\gamma_{\text {in }}$ & 0.543 & 0.543 & 0 \\
$\gamma_{\text {out }}$ & 0.6 & 0.6 & 0 \\
\hline \hline
\end{tabular}

\begin{tabular}{|l|c|c|c|c|}
\hline \multirow{2}{*}{} & \multicolumn{2}{|c|}{$\varepsilon_{\text {post-iDM }}^{\text {total }}$} & \multicolumn{2}{c|}{$\varepsilon_{\text {block }}$} \\
\cline { 2 - 5 } & Sim. & Est. & Sim. & Est. \\
\hline CCDM & $\circ$ & - & $\bullet$ & $\cdots \cdot \cdot$ \\
\hline HiDM & $\square$ & $--\cdot$ & & ---- \\
\hline BICM & $\Delta$ & -- & $\Delta$ & $-\cdot-$ \\
\hline
\end{tabular}

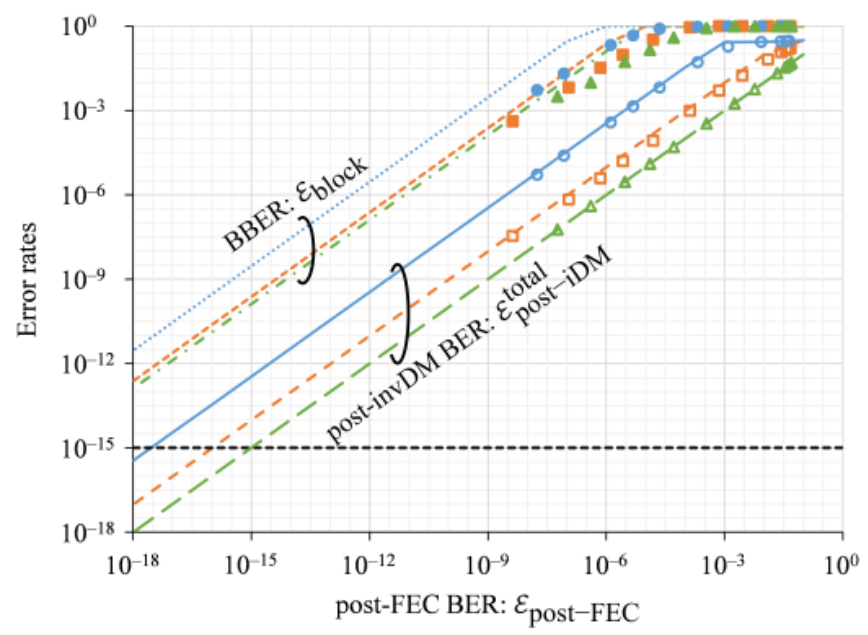

Fig. 7. Post-invDM BER from simulation or estimation under the assumption of a single-error input to the invDM per DM word.

FEC codeword is larger than 1 due to the bursty nature of FEC decoders, $\mathcal{E}_{\text {FEC-fr }}$ becomes closer to $\mathcal{E}_{\text {post-FEC }}$. The quantity $N_{\mathrm{u}} /$ $\left(R_{\mathrm{c}} m N_{\mathrm{s}} / 2\right)$ in 16$)$ is 0.875 for CCDM/HiDM-based PS-256QAM and 1 for BICM-based 128-QAM.

The ratio $r_{\mathcal{E} 1}$ can be calculated based on (12) and (13), which gives $r_{\mathcal{E} 1}=348$ and 9.7 for CCDM and HiDM, resp. These theoretic results are of the same order as the simulation results in the previous section. This single-error scenario gives the largest $r_{\mathcal{E} 1}$ (the post-invDM BER would be worse than the case with burst errors).

In Fig. 7, we estimated the post-invDM BER and BBER as a function of the post-FEC BER at low error rates using the bounds 13 and 16 . The post-invDM BER $\mathcal{E}_{\text {post-iDM }}^{\text {total }}$ estimates agree well with the simulation results. To achieve a post-invDM BER of $10^{-15}$, a post-FEC BER of $3 \cdot 10^{-18}$ (for CCDM) or $10^{-16}$ (for HiDM) is required. We can thus conclude that the FEC design and evaluation becomes more difficult with reverse concatenation PS than with BICM, due to the requirement of such low post-FEC BERs. If the code would have an error floor just below a post-FEC BER of $10^{-15}$, the required SNR for a post-invDM BER of $10^{-15}$ would be significantly larger. Also, simulations to evaluate a post-FEC BER of $3 \cdot 10^{-18}$ to ensure that there is no error floor are very challenging (to transmit $10^{18}$ bits at $100 \mathrm{Gbit} / \mathrm{s}$ takes 
$10^{7} \mathrm{~s}$, or 3.8 months). HiDM requires an order of magnitude higher error rate than CCDM, so the gap between BICM and reverse concatenation PS is greatly reduced. As for the BBER $\mathcal{E}_{\text {block}}$, estimations and simulations approach each other in the low post-FEC BER regime, where the single-error assumption works well. The bounded BBER as a function of the post-FEC BER for HiDM is almost the same as for BICM-based 128QAM. The BBER bound for HiDM is 16 times lower than for CCDM in the case of the same post-FEC BER, because the HiDM places the DM word on the local area due to the high throughput feature compared with the CCDM, as shown in Fig. 3. When the DM word length $N_{\mathrm{s}}$ is increased, the postFEC error rates are reduced, but the bounds on $\mathcal{E}_{\text {post-iDM }}^{\text {sb }}$ and $\mathcal{E}_{\text {block }}$ in 12 and $(16)$ increase proportionally to $N_{\mathrm{s}}$.

\section{SUMMARY AND OUTLOOK}

We proposed HiDM for low-complexity implementation of reverse-concatenation PS, and evaluated its end-to-end performance for high-throughput fiber-optic communications. As the post-invDM BER and BBER performance depends on the FEC decoding, invDM processing, and cross-layout of the FEC codewords and the DM words, we demonstrated frame structure examples considering the combined FEC and PS. By a back-to-back error insertion test and a single-error assumption, very low post-invDM error rates can be estimated from the post-FEC BER, which allows relaxed requirements for the post-FEC BER if HiDM is used instead of CCDM. The combination of known post-FEC BER prediction techniques using NGMI or ASI with the proposed post-invDM error rate estimation from post-FEC BER would be an interesting topic for further studies. Other future research directions include a careful design of DM/invDM algorithms considering the implementation combined with FEC to reach the desired BER and BBER performance targets.

\section{ACKNOWLEDGMENTS}

We thank Alex Alvarado of Eindhoven University of Technology, David S. Millar of Mitsubishi Electric Research Laboratories, and Koji Igarashi of Osaka University for fruitful discussions about performance metrics, distribution matching, and reverse concatenation.

\section{REFERENCES}

[1] F. Chang, K. Onohara, and T. Mizuochi, "Forward error correction for $100 \mathrm{G}$ transport networks," IEEE Commun. Mag., vol. 48, no. 3, pp. S48-S55, Mar. 2010.

[2] E. Agrell and M. Secondini, "Information-theoretic tools for optical communications engineers," to appear in Proc. IEEE Phot. Conf. (IPC), Reston, VA, Sep.-Oct. 2018.

[3] G. D. Forney, Jr. and L.-F. Wei, "Multidimensional constellations-Part I: introduction, figure of merit, and generalized cross constellation," IEEE J. Selected Areas Commun., vol. 7, no. 6, pp. 877-892, Aug. 1989.

[4] A. R. Calderbank and L. H. Ozarow, "Nonequiprobable signaling on the Gaussian channel," IEEE Trans. Inf. Theory, vol. 36, no. 4, pp. 726-740, July 1990.

[5] F. R. Kschischang and S. Pasupathy, "Optimal nonuniform signaling for Gaussian channels," IEEE Trans. Inf. Theory, vol. 39, no. 3, pp. 913929, May 1993.

[6] D. Raphaeli and A. Gurevitz, "Constellation shaping for pragmatic turbo-coded modulation with high spectral efficiency," IEEE Trans. Commun., vol. 52, no. 3, pp. 341-345, Mar. 2004.
[7] K. Roberts, M. O’Sullivan, K.-T. Wu, H. Sun, A. Awadalla, D. J. Krause, and C. Laperle, "Performance of dual-polarization QPSK for optical transport systems," J. Lightw. Technol., vol. 27, no. 16, pp. 3546-3559, Aug. 2009.

[8] E. Agrell and M. Karlsson, "Power-efficient modulation formats in coherent transmission systems," J. Lightw. Technol., vol. 27, no. 22, pp. 5115-5126, Nov. 2009.

[9] R. Dar, M. Feder, A. Mecozzi, and M. Shtaif, "On the shaping gain in the nonlinear fiber-optic channel," in Proc. IEEE Int. Symp. Inf. Theory, pp. 2794-2798, July 2014.

[10] D. S. Millar, T. Koike-Akino, S. Ö. Arık, K. Kojima, K. Parsons, T. Yoshida, and T. Sugihara, "High-dimensional modulation for coherent optical communications systems," Opt. Exp., vol. 22, no. 7, pp. 87988812, Apr. 2014.

[11] A. D. Shiner, M. Reimer, A. Borowiec, S. O. Gharan, J. Gaudette, P. Mehta, D. Charlton, K. Roberts, and M. O'Sullivan, "Demonstration of an 8-dimensional modulation format with reduced inter-channel nonlinearities in a polarization multiplexed coherent system," Opt. Exp., vol. 22, no. 17, pp. 20366-20374, Aug. 2014.

[12] K. Kojima, T. Yoshida, T. Koike-Akino, D. S. Millar, K. Parsons, M. Pajovic, and V. Arlunno, "Nonlinearity-tolerant four-dimensional 2A8PSK family for 5-7 bits/symbol spectral efficiency," J. Lightw. Technol., vol. 35, no. 8, pp. 1383-1391, Apr. 2017.

[13] L. Beygi, E. Agrell, J. M. Kahn, and M. Karlsson, "Rate-adaptive coded modulation for fiber-optic communications," J. Lightw. Technol., vol. 32, no. 2, pp. 333-343, Jan. 2014

[14] M. P. Yankov, F. D. Ros, E. P. da Silva, S. Forchhammer, K. J. Larsen, L. K. Oxenløwe, M. Galili, and D. Zibar, "Constellation shaping for WDM systems using 256QAM/1024QAM with probabilistic optimization," J. Lightw. Technol., vol. 34, no. 22, pp. 5146-5156, Nov. 2016.

[15] G. Böcherer, F. Steiner, and P. Schulte, "Bandwidth efficient and rate-matched low-density parity-check coded modulation," IEEE Trans. Commun., vol. 63, no. 12, pp. 4651-4665, Dec. 2015

[16] W. G. Bliss, "Circuitry for performing error correction calculations on baseband encoded data to eliminate error propagation," IBM Techn. Discl. Bul., vol. 23, pp. 4633-4634, 1981.

[17] J. L. Fan and J. M. Cioffi, "Constrained coding techniques for soft iterative decoders," in Proc. Global Telecommunications Conference (GLOBECOM), Rio de Janeiro, Brazil, Dec. 1999, vol. 1(B), pp. 723727.

[18] I. B. Djordjevic and B. V. Vasic, "Constrained coding techniques for the suppression of intrachannel nonlinear effects in high-speed optical transmission," J. Lightw. Technol., vol. 24, no. 1, pp. 411-419, Jan. 2006.

[19] L. Szczecinski and A. Alvarado, Bit-Interleaved Coded Modulation: Fundamentals, Analysis, and Design. New York, NY, USA: Wiley, 2015.

[20] P. Schulte and G. Böcherer, "Constant composition distribution matching,” IEEE Trans. Inf. Theory, vol. 62, no. 1, pp. 430-434, Jan. 2016.

[21] F. Buchali, F. Steiner, G. Böcherer, L. Schmalen, P. Schulte, and W. Idler, "Rate adaptation and reach increase by probabilistically shaped 64 QAM: an experimental demonstration," J. Lightw. Technol., vol. 34, no. 7, pp. 1599-1609, Apr. 2016.

[22] T. Yoshida, M. Karlsson, and E. Agrell, "Technologies toward implementation of probabilistic constellation shaping," in Proc. Eur. Conf. on Opt. Comm. (ECOC), Roma, Italy, Sep. 2018, p. Th.1.H.1.

[23] ITU-T, "Interfaces for the optical transport network," 2016. [Online]. Available: www.itu.int/rec/T-REC-G.709

[24] ITU-T, "Error performance parameters and objectives for multioperator international paths within optical transport networks," 2011. [Online]. Available: www.itu.int/rec/T-REC-G.8201

[25] ITU-T, "Forward error correction for high bit-rate DWDM submarine systems," 2004. [Online]. Available: www.itu.int/rec/T-REC-G.975.1

[26] L. Schmalen, A. Alvarado, R. Rios-Müller, "Performance prediction of nonbinary forward error correction in optical transmission experiments," J. Lightw. Technol., vol. 35, no. 4, pp. 1015-1027, Feb. 2017.

[27] A. Alvarado, E. Agrell, D. Lavery, R. Maher, and P. Bayvel, "Replacing the soft FEC limit paradigm in the design of optical communication systems," J. Lightw. Technol., vol. 33, no. 20, pp. 4338-4352, Oct. 2015.

[28] G. Böcherer, "Achievable rates for probabilistic shaping," [Online]. Available: arxiv.org/abs/1707.01134

[29] J. Cho, L. Schmalen, and P. J.Winzer, "Normalized generalized mutual information as a forward error correction threshold for probabilistically shaped QAM," in Proc. Eur. Conf. Opt. Commun. (ECOC), Göteborg, Sweden, Sep. 2017, p. M.2.D.2.

[30] T. Yoshida, M. Karlsson, and E. Agrell, "Performance metrics for systems with soft-decision FEC and probabilistic shaping," Photon. Technol. Lett., vol. 29, no. 23, pp. 2111-2114, Dec. 2017. 
[31] T. Fehenberger, D. S. Millar, T. Koike-Akino, K. Kojima, and K. Parsons, "Multiset-partition distribution matching," IEEE Trans. Commun., to appear, DOI 10.1109/TCOMM.2018.2881091.

[32] Y. C. Gültekin, W. van Houtum, and F. Willems, "On constellation shaping for short block lengths," in Proc. Symp. on Inf. Theory and Signal Process in the Benelux (SITB), June 2018.

[33] Y. C. Gültekin, F. M. J. Willems, W. J. Houtum, S. Şerbetli, "Constellation shaping for IEEE 802.11," in Proc. IEEE 28th Annual Int. Symp. on Personal, Indoor, and Mobile Radio Communications (PIMRC), Montreal, Canada, Oct. 2017.

[34] Y. C. Gültekin, F. M. J. Willems, W. J. Houtum, S. Şerbetli, "Approximate enumerative sphere shaping," in Proc. IEEE Int. Symp. Inf. Theory, Vail, CO, USA, June 2018, pp. 676-680.

[35] P. Schulte and F. Steiner, "Divergence-optimal fixed-to-fixed length distribution matching with shell mapping," [Online]. Available: www. arxiv.org/pdf/1803.03614v2

[36] T. Yoshida, M. Karlsson, and E. Agrell, "Post-FEC BER prediction accuracy for probabilistically shaped signaling in fiber-optic communications," in Proc. Eur. Conf. on Opt. Comm. (ECOC), Göteborg, Sweden, Sep. 2017, p. Mo.2.D.3.

[37] T. V. Ramabadran, "A coding scheme for $m$-out-of- $n$ codes," IEEE Trans. Commun., vol. 38, no. 8, pp. 1156-1163, Aug. 1990.

[38] J. Cho, S. Chandrasekhar, R. Dar, and P. J. Winzer, "Low-complexity shaping for enhanced nonlinearity tolerance," in Proc. Eur. Conf. on Opt. Comm. (ECOC), Düsseldorf, Germany, Sep. 2016, p. W1C.2.

[39] T. Yoshida, M. Karlsson, and E. Agrell, "Short-block-length shaping by simple mark ratio controllers for granular and wide-range spectral efficiencies," in Proc. Eur. Conf. on Opt. Comm. (ECOC), Göteborg, Sweden, Sep. 2017, p. Tu.2.D.2.

[40] G. Böcherer, F. Steiner, and P. Schulte, "Fast probabilistic shaping implementation for long-haul fiber-optic communication systems," in Proc. Eur. Conf. on Opt. Comm. (ECOC), Göteborg, Sweden, Sep. 2017, p. Tu.2.D.3.

[41] T. Yoshida, M. Karlsson, and E. Agrell, "Low-complexity variablelength output distribution matching with periodical distribution uniformalization," in Proc. Opt. Fib. Commun. Conf. (OFC), San Diego, CA, USA, Mar. 2018, p. M.4.E.2.

[42] M. Pikus and W. Xu, "Bit-level probabilistically shaped coded modulation,", IEEE Commun. Lett., vol. 21, no. 9, pp. 1929-1932, Sep. 2017.

[43] G. Böcherer, F. Steiner, and P. Schulte, "High throughput probabilistic shaping with product distribution matching," [Online]. Available: www. arxiv.org/pdf/1702.07510v4

[44] F. Steiner, P. Schulte, and G. Böcherer. "Approaching waterfilling capacity of parallel channels by higher order modulation and probabilistic amplitude shaping," in Proc. 52nd Annual Conference on Information Sciences and Systems (CISS), Princeton, NJ, USA, Mar. 2018.

[45] D. Che, W. Shieh, "Approaching the capacity of colored-SNR optical channels by multicarrier entropy loading," J. Lightw. Technol., vol. 36, no. 1, pp. 68-78, Jan. 2018.

[46] European Telecommunications Standards Institute, "Second generation framing structure, channel coding and modulation systems for broadcasting, interactive services, news gathering and other broadband satellite applications; Part 1 (DVB-S2)," ETSI Standard EN 302 307-1 V1.4.1, Nov. 2014. [Online]. Available: www.dvb.org/standards

[47] R. Mahadevappa, S. ten Brink, "Comparison of 128QAM mappings/labelings for 802.11n," IEEE, 802.11-03/825r0, Nov. 2003. 